\title{
Implications of differences in expression of sarcosine metabolism-related proteins according to the molecular subtype of breast cancer
}

Ja Kyung Yoon, Do Hee Kim and Ja Seung Koo*

\begin{abstract}
Background: The goal of this study was to investigate the expression of sarcosine metabolism-related proteins, namely glycine $\mathrm{N}$-methyltransferase (GNMT), sarcosine dehydrogenase (SARDH), and I-pipecolic acid oxidase (PIPOX), in the different breast cancer subtypes and to assess the implications of differences in expression pattern according to subtype.
\end{abstract}

Methods: We analyzed the expression of GNMT, SARDH, and PIPOX in a tissue microarray of 721 breast cancer cases using immunohistochemistry (IHC). We classified breast cancer cases into subtype luminal A, luminal B, HER-2, and triple negative breast cancer (TNBC) according to the status for the estrogen receptor (ER), progesterone receptor (PR), HER-2, and Ki-67. Sarcosine metabolism phenotype was stratified according to IHC results for GNMT, SARDH, and PIPOX: GNMT(+), SARDH and PIPOX(-) was classified as high sarcosine type; GNMT(-), SARDH or PIPOX(-) as low sarcosine type; GNMT(+), SARDH or PIPOX(+) as intermediate sarcosine type, and GNMT(-), SARDH and PIPOX(-) as null type.

Results: Expression of sarcosine metabolism-related proteins differed significantly according to breast cancer subtype (GNMT, $p=0.005$; SARDH, $p=0.012$; tumoral PIPOX, $p=0.008$; stromal PIPOX, $p<0.001$ ). These proteins were the most frequently expressed in HER-2 type tumors and the least in TNBC. Sarcosine metabolism phenotype also varied according to breast cancer subtype, with high sarcosine type the most common in HER-2, and null type the most common in TNBC $(p=0.003)$. Univariate analysis revealed that GNMT expression $(p=0.042)$, tumoral PIPOX negativity $(p=0.039)$, and high sarcosine type $(p=0.021)$ were associated with shorter disease-free survival (DFS). Multivariate analysis also revealed GNMT expression was an independent factor for shorter DFS (hazard ratio: 2.408, 95\% Cl: 1.154-5.024, $p=0.019$ ).

Conclusion: Expressions of sarcosine metabolism-related proteins varied according to subtype of breast cancer, with HER-2 type tumors showing elevated expression of these proteins, and TNBC subtype showing decreased expression of these proteins. Expression of sarcosine metabolism-related proteins was also associated with breast cancer prognosis.

Keywords: Breast cancer, Metabolism, Molecular subtype, Sarcosine

\footnotetext{
* Correspondence: kjs1976@yuhs.ac

Department of Pathology, Yonsei University College of Medicine, Severance

Hospital, 50 Yonsei-ro, Seodaemun-gu, Seoul, South Korea
} 


\section{Background}

Sarcosine (N-methylglycine), a non-proteogenic amino acid produced in the synthesis and degradation of glycine, is produced by methyl group transfer from $S$-adenosylmethionine (SAM) to glycine by glycine $N$-methyltransferase (GNMT). Other major sarcosine-metabolizing enzymes sarcosine dehydrogenase (SARDH) and I-pipecloioc acid oxidase (PIPOX) - detach the methyl group from sarcosine via oxidative demethylation to form glycine [1]. The role of sarcosine extends beyond its identity as a non-proteogenic amino acid; it is also a potential oncometabolite. Prostate cancer studies have reported that sarcosine is a sensitive tumor biomarker and suggest its involvement in tumor progression and metastatic processes [2-4].

Breast cancer is clinically, histopathologically, and molecularly heterogeneous. Efforts to classify tumors with similar characteristics have resulted in subtyping of breast cancer into luminal A, luminal B, HER-2, normal breast-like, and basal-like types through genetic profiling analysis $[5,6]$. A separate set of subtyping criteria relies on the expression of important therapeutic markers: estrogen receptor (ER), progesterone receptor (PR), and HER-2, from which the term triple negative breast cancer (TNBC) is derived. TNBC is defined as breast cancer negative for all three markers, and a significant overlap of biological and clinical characteristics between TNBC and basal-like breast cancers, resulting in more than $85 \%$ of TNBC belong to basal-like breast cancer [7]. These molecular differences are associated with histopathological and clinical differences as well as variations in treatment response and prognosis, implicating possible differences in metabolic features. Previous studies indicate elevated expression of the glycolysisrelated proteins GLUT-1 and CAIX in basal-like type/ TNBC breast cancers $[8,9]$ and elevated expression of the glutaminolysis-related protein in HER-2 type breast cancers [10], supporting a plausible relation between metabolism and molecular subtype. However, the association between breast cancer subtype and sarcosine metabolism-related protein expression has not been examined. While most research on sarcosine up to date has been done in prostate cancer, there exists considerable similarity and connection between prostate cancer and breast cancer. Firstly, epidemiologic studies show that family history of breast cancer significantly influences the risk for prostate cancer $[11,12]$. Secondly, genetic studies showed that androgen receptor(AR) alteration, which is important in the development of prostate cancer, is also present in breast cancer [13]. Moreover, studies have shown that the mutations significant for hereditary breast cancer, BRCA1 and BRCA2 mutations, is related to higher risk for prostate cancer $[14,15]$. Lastly, in a biochemical aspect, the prostate cancer-sensitive marker PSA was also detected in breast cancer [16], and its relation to good prognosis of prostate cancer was reported [17]. Conclusively, the epidemiologic, genetic and biochemical similarities suggest a resemblance in sarcosine metabolism between prostate cancer and breast cancer.

Therefore, in this study, we determined the expression of sarcosine metabolism-related protein in various breast cancer subtypes and investigated the implications of differences in expression pattern according to subtype.

\section{Materials and methods Patient selection}

Subjects were selected from among patients diagnosed with invasive breast cancer who received surgical treatment from January 2000 to December 2006 at Severance Hospital. Patients who received pre-operative hormone or chemotherapy were excluded. This study was approved by the Institutional Review Board of Yonsei University Severance Hospital. IRB exempted the informed consent from patients. All cases were reviewed retrospectively by a breast pathologist (Koo JS) using Hematoxylin \& Eosin (H\&E)-stained slides. Histological grade was assessed using the Nottingham grading system [18]. Clinicopathologic parameters evaluated in each case included patient age at initial diagnosis, lymph node metastasis, tumor recurrence, distant metastasis, and patient survival.

\section{Tissue microarray}

A representative area was selected on an H\&E-stained slide, and a corresponding spot was marked on the surface of the paraffin block. Using a biopsy needle, the selected area was punched out, and a 3-mm tissue core was transferred to a $6 \times 5$ recipient block. Two tissue cores of invasive tumor were extracted to minimize extraction bias. Each tissue core was assigned a unique tissue microarray location number that was linked to a database containing other clinicopathologic data.

\section{Immunohistochemistry}

Antibodies used for immunohistochemistry are listed in Additional file 1: Table S1. All immunohistochemistry was performed with formalin-fixed, paraffin-embedded tissue sections. Briefly, 5 - $\mu$ m-thick sections were obtained with a microtome, transferred onto adhesive slides, and dried at $62^{\circ} \mathrm{C}$ for 30 minutes. After incubation with primary antibodies, immunodetection was performed with biotinylated anti-mouse immunoglobulin, followed by peroxidase-labeled streptavidin using a labeled streptavidin biotin kit with 3,3'-diaminobenzidine chromogen as the substrate. The primary antibody incubation step was omitted in the negative control. Positive control tissue was used as per the manufacturer's recommendation. Slides were counterstained with Harris hematoxylin. 


\section{Interpretation of immunohistochemical staining}

All immunohistochemical markers were accessed by light microscopy. A cut-off value of $1 \%$ or more positively stained nuclei was used to define ER and PR positivity [19]. HER-2 staining was analyzed according to the American Society of Clinical Oncology (ASCO)/College of American Pathologists (CAP) guidelines using the following categories: $0=$ no immunostaining; $1+=$ weak incomplete membranous staining, less than $10 \%$ of tumor cells; $2+=$ complete membranous staining, either uniform or weak in at least $10 \%$ of tumor cells; and $3+=$ uniform intense membranous staining in at least $30 \%$ of tumor cells [20]. HER-2 immunostaining was considered positive when strong $(3+)$ membranous staining was observed, whereas cases with 0 to $1+$ were regarded as negative. Cases showing 2+ HER-2 expression were evaluated for
HER-2 amplification by fluorescent in situ hybridization (FISH).

Immunohistochemical markers for GNMT, SARDH, and PIPOX were accessed by light microscopy. Immunohistochemical staining was calculated as the product of the proportion of stained cells and immunostaining intensity. Proportion of stained cells was stratified as 0 : negative, 1: less than $30 \%$ positive, and 2: equal to or more than $30 \%$ positive, while immunostaining intensity was stratified as 0: negative, 1: weak, 2: moderate, and 3: strong. Immunohistochemistry was deemed negative when the product of the proportion of stained cells and immunostaining intensity was $0-1$ and positive when the product was 2-6 [21]. Ki-67 labeling index (LI) was defined as the percentage of cancer cells with a Ki-67positive nucleus.

Table 1 Clinicopathologic characteristics of patients according to breast cancer phenotype

\begin{tabular}{|c|c|c|c|c|c|c|}
\hline Parameter & $\begin{array}{l}\text { Total } \\
(\mathrm{n}=721)(\%) \\
\end{array}$ & $\begin{array}{l}\text { Luminal A } \\
(n=303)(\%)\end{array}$ & $\begin{array}{l}\text { Luminal B } \\
(\mathrm{n}=169)(\%)\end{array}$ & $\begin{array}{l}\text { HER-2 } \\
(\mathrm{n}=71)(\%)\end{array}$ & $\begin{array}{l}\text { TNBC } \\
(n=178)(\%)\end{array}$ & $P$-value \\
\hline Age (years) & & & & & & 0.012 \\
\hline$\leq 50$ & $428(59.4)$ & $173(57.1)$ & $113(66.9)$ & $32(45.1)$ & $110(61.8)$ & \\
\hline$>50$ & $293(40.6)$ & $130(42.9)$ & $56(33.1)$ & $39(54.9)$ & $68(38.2)$ & \\
\hline Histologic grade & & & & & & $<0.001$ \\
\hline$|/| \mid$ & $485(67.3)$ & $275(90.8)$ & $112(66.3)$ & $37(52.1)$ & $61(34.3)$ & \\
\hline III & $236(32.7)$ & $28(9.2)$ & $57(33.7)$ & $34(47.9)$ & $117(65.7)$ & \\
\hline Tumor stage & & & & & & 0.002 \\
\hline $\mathrm{T} 1$ & $355(49.2)$ & $169(55.8)$ & $86(50.9)$ & $32(45.1)$ & $68(38.2)$ & \\
\hline $\mathrm{T} 2 / \mathrm{T} 3$ & $366(50.8)$ & $134(44.2)$ & $83(49.1)$ & $39(54.9)$ & $110(61.8)$ & \\
\hline Nodal metastasis & & & & & & 0.162 \\
\hline Absent & $427(59.2)$ & $173(57.1)$ & $93(55.0)$ & $44(62.0)$ & $117(65.7)$ & \\
\hline Present & $294(40.8)$ & $130(42.9)$ & $76(45.0)$ & $27(38.0)$ & $61(34.3)$ & \\
\hline Estrogen receptor status & & & & & & $<0.001$ \\
\hline Negative & $259(35.9)$ & $5(1.7)$ & $5(3.0)$ & $71(100.0)$ & $178(100.0)$ & \\
\hline Positive & $462(64.1)$ & $298(98.3)$ & $164(97.0)$ & $0(0.0)$ & $0(0.0)$ & \\
\hline Progesterone receptor status & & & & & & $<0.001$ \\
\hline Negative & $347(48.1)$ & $50(16.5)$ & $48(28.4)$ & $71(100.0)$ & $178(100.0)$ & \\
\hline Positive & $374(51.9)$ & $253(83.5)$ & $121(71.6)$ & $0(0.0)$ & $0(0.0)$ & \\
\hline HER-2 status & & & & & & $<0.001$ \\
\hline Negative & $565(78.4)$ & $303(100.0)$ & $84(49.5)$ & $0(0.0)$ & $178(100.0)$ & \\
\hline Positive & $156(21.6)$ & $0(0.0)$ & $85(50.3)$ & $71(100.0)$ & $0(0.0)$ & \\
\hline Ki-67 LI (\%) & & & & & & $<0.001$ \\
\hline$\leq 14$ & $409(56.7)$ & $303(100.0)$ & $49(29.0)$ & $29(40.8)$ & $28(15.7)$ & \\
\hline$>14$ & $312(43.3)$ & $0(0.0)$ & $120(71.0)$ & $42(59.2)$ & $150(84.3)$ & \\
\hline Tumor recurrence & $63(8.7)$ & $15(5.0)$ & $13(7.7)$ & $11(15.5)$ & $24(13.5)$ & 0.002 \\
\hline No. of patient deaths & $61(8.5)$ & $13(4.3)$ & $13(7.7)$ & $11(15.5)$ & $24(13.5)$ & 0.001 \\
\hline Duration of clinical follow-up (months, mean \pm SD) & $69.9 \pm 31.2$ & $71.9 \pm 29.0$ & $70.0 \pm 30.1$ & $66.1 \pm 34.9$ & $68.1 \pm 34.1$ & 0.419 \\
\hline
\end{tabular}

Bold number represents $\mathrm{p}<0.05$.

$\mathrm{TNBC}$, triple negative breast cancer. 
Table 2 Expression of metabolism-related proteins according to breast cancer subtype

\begin{tabular}{|c|c|c|c|c|c|c|}
\hline Parameter & Total $(\mathrm{n}=721)(\%)$ & Luminal A ( $n=303)(\%)$ & Luminal B ( $n=169)(\%)$ & HER-2 $(n=71)(\%)$ & TNBC $(n=178)(\%)$ & $P$-value \\
\hline GNMT & & & & & & 0.005 \\
\hline Negative & $664(92.1)$ & $273(90.1)$ & $153(90.5)$ & $63(88.7)$ & $175(98.3)$ & \\
\hline Positive & $57(7.9)$ & $30(9.9)$ & $16(9.5)$ & $8(11.3)$ & $3(1.7)$ & \\
\hline SARDH & & & & & & 0.012 \\
\hline Negative & $597(82.8)$ & $261(86.1)$ & $130(76.9)$ & $53(74.6)$ & $153(86.0)$ & \\
\hline Positive & $124(17.2)$ & $42(13.9)$ & $39(23.1)$ & $18(25.4)$ & $25(14.0)$ & \\
\hline PIPOX (T) & & & & & & 0.008 \\
\hline Negative & $570(79.1)$ & $225(74.3)$ & $143(84.6)$ & $52(73.2)$ & $150(84.3)$ & \\
\hline Positive & $151(20.9)$ & $78(25.7)$ & $26(15.4)$ & $19(26.8)$ & $28(15.7)$ & \\
\hline PIPOX (S) & & & & & & $<0.001$ \\
\hline Negative & $671(93.1)$ & $287(94.7)$ & $154(91.1)$ & $58(81.7)$ & $172(96.6)$ & \\
\hline Positive & $50(6.9)$ & $16(5.3)$ & $15(8.9)$ & $13(18.3)$ & $6(3.4)$ & \\
\hline
\end{tabular}

\section{FISH analysis}

Before FISH analysis, invasive tumors were examined on hematoxylin-eosin stained slides. FISH was subsequently performed on the tested tumor using a PathVysion HER-2 DNA Probe Kit (Vysis, Downers Grove, IL, USA) in accordance with the manufacturer's instructions. HER-2 gene copy number was evaluated using an epifluorescence microscope (Olympus, Tokyo, Japan). At least 60 tumor cell nuclei in three separate regions were investigated for HER-2 and chromosome 17 signals. HER-2 gene amplification was determined according to the ASCO/CAP guidelines [20]. An absolute HER-2 gene copy number less than 4 or a HER-2 gene/chromosome 17 copy number ratio (HER-2/Chr17 ratio) less than 1.8 was considered $H E R-2$ negative. An absolute $H E R-2$ copy number between 4 and 6 or a HER-2/Chr17 ratio between 1.8 and 2.2 was considered HER-2 equivocal. An absolute HER-2 copy number greater than 6 or a HER-2/Chr17 ratio higher than 2.2 was considered HER-2 positive.

\section{Tumor phenotype classification}

In this study, we classified breast cancer phenotypes according to the immunohistochemistry results for ER,

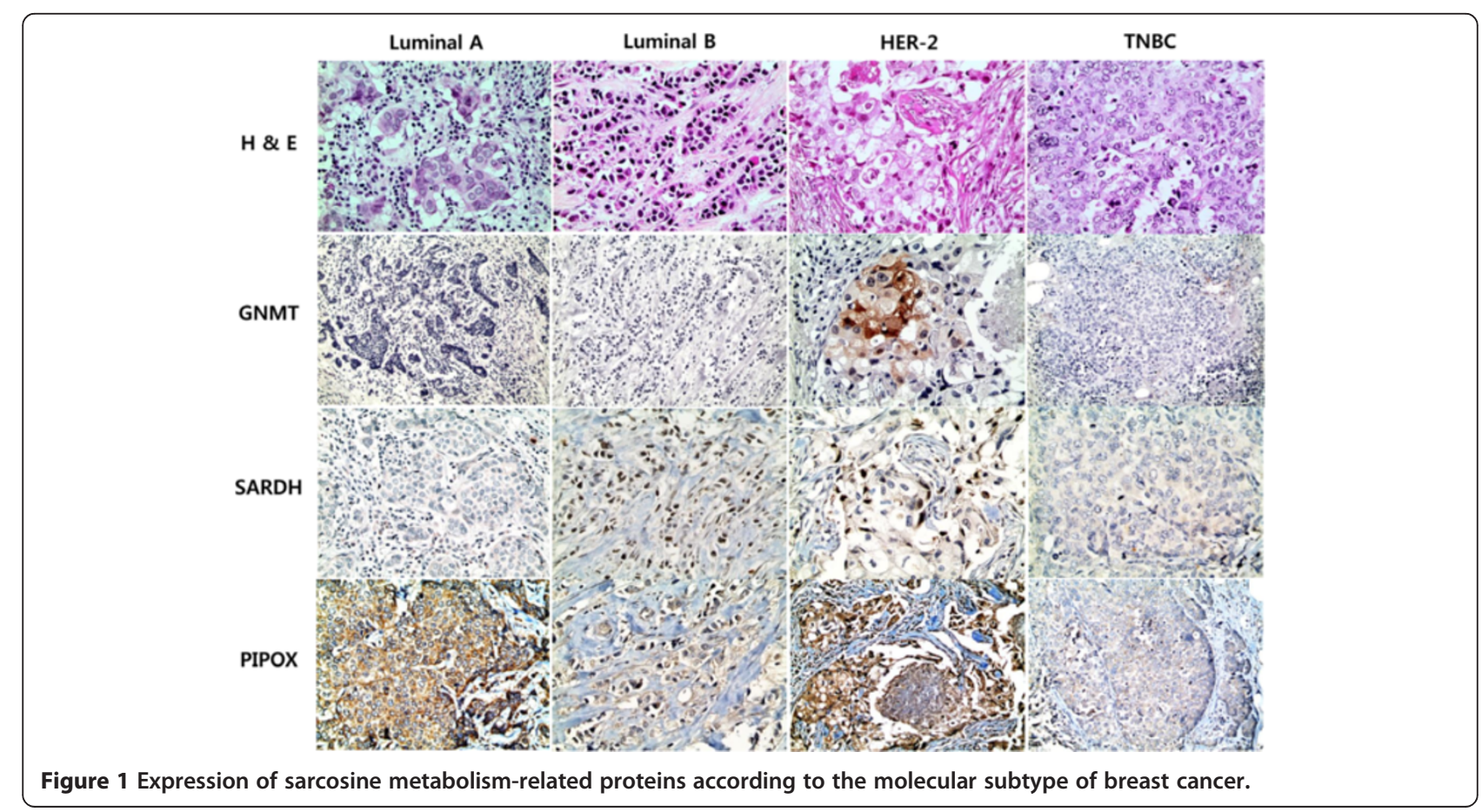




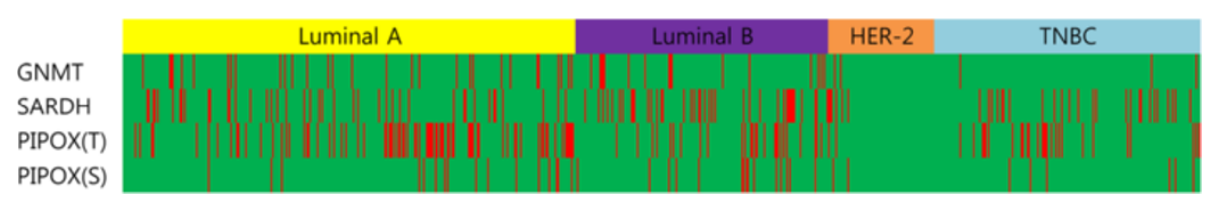

Figure 2 A heatmap of the expression of sarcosine metabolism-related proteins according to the molecular subtype of breast cancer. T, tumor, S, stroma, red: positive, green: negative.

PR, HER-2, Ki-67 and FISH results for HER-2 as follows [22]: luminal A type, ER or/and PR positive, HER-2 negative and Ki-67 LI <14\%; Luminal B type, (HER-2 negative) ER or/and PR positive, HER-2 negative and Ki-67 LI $\geq 14 \%$; (HER-2 positive) ER or/and PR positive and HER-2 overexpressed or/and amplified; HER-2 overexpression type, ER and PR negative and HER-2 overexpressed or/and amplified; TNBC type: ER, PR, and HER-2 negative.

\section{Sarcosine metabolism phenotype}

Sarcosine metabolism phenotype was classified according to immunohistochemistry results for GNMT, SARDH, and PIPOX. High sarcosine type was defined as GNMT $(+) /$ SARDH and PIPOX $(-)$, low sarcosine type was defined as GNMT(-)/SARDH or PIPOX(+), intermediate sarcosine type was defined as GNMT(+)/SARDH or $\operatorname{PIPOX}(+)$, and null type was defined as GNMT $(-) / S A R D H$ and PIPOX $(-)$.

\section{Statistical analysis}

Data were analyzed using SPSS for Windows, Version 12.0 (SPSS Inc., Chicago, IL, USA). For determination of statistical significance, Student's $t$ and Fisher's exact tests were used for continuous and categorical variables, respectively. In the case of analyzing data with multiple comparisons, a corrected p-value with the application of the Bonferroni multiple comparison procedure was used. Statistical significance was set to $\mathrm{p}<0.05$. Kaplan-Meier survival curves and log-rank statistics were employed to evaluate time to tumor recurrence and overall survival. Multivariate regression analysis was performed using the Cox proportional hazards model.

\section{Results}

\section{Patient clinicopathologic characteristics}

Of a total of 721 subjects, there were 303 (42.0\%) luminal A, 169 (23.4\%) luminal B, 71 (9.8\%) HER-2 type, and 178 (24.7\%) TNBC. Clinicopathologic analysis revealed that TNBC tumors had a higher histological grade $(\mathrm{p}<0.001)$, higher T stage $(\mathrm{p}=0.002)$, and higher Ki-67 LI $(\mathrm{p}<0.001)$ than the other tumor sub-types. In contrast, the HER-2 type was associated with older patient age $(\mathrm{p}=0.012)$, higher tumor recurrence rate $(p=0.002)$, and higher mortality $(\mathrm{p}=0.001)$ than the other subtypes (Table 1$)$.

\section{Expression of sarcosine metabolism-related proteins according to tumor phenotype}

Analysis of sarcosine metabolism-related protein expression revealed differences in expression depending on molecular subtype. HER-2 type tumors the most frequently showed the expression of GNMT $(\mathrm{p}=0.005)$, SARDH $(\mathrm{p}=0.012)$, tumoral PIPOX $(\mathrm{p}=0.008)$, and stromal PIPOX $(p<0.001)$, while TNBC tumors the least frequently exhibited the expression of all four proteins (Table 2, Figure 1, and Figure 2). Sarcosine metabolism phenotypes also varied according to molecular subtype; HER-2 type tumors had the highest ratio of high sarcosine type, while TNBC tumors had the highest ratio of null type ( $p=0.003$, Table 3 ). Clinically, there were statistically significant differences in ER expression ( $p=0.049)$, PR expression ( $\mathrm{p}=0.011)$, Ki-67 LI ( $\mathrm{p}=0.007)$, and tumor recurrence $(p=0.022)$ according to sarcosine metabolism type. Intermediate sarcosine type had the highest rate of ER and PR positivity with a low Ki-67 LI, while null type tumors had the lowest ER and PR positivity with a high Ki-67 LI. High sarcosine type showed the highest tumor recurrence rate (Table 4).

Table 3 Sarcosine metabolism phenotypes of different breast cancer subtypes

\begin{tabular}{|c|c|c|c|c|c|c|}
\hline Parameter & $\begin{array}{l}\text { Total } \\
(\mathrm{N}=721)(\%)\end{array}$ & $\begin{array}{l}\text { Luminal A } \\
(n=303)(\%)\end{array}$ & $\begin{array}{l}\text { Luminal B } \\
(n=169)(\%)\end{array}$ & $\begin{array}{l}\text { HER-2 } \\
(n=71)(\%)\end{array}$ & $\begin{array}{l}\text { TNBC } \\
(n=178)(\%)\end{array}$ & $P$-value \\
\hline Sarcosine metabolic type & & & & & & 0.003 \\
\hline High sarcosine type & $36(5.0)$ & $16(5.3)$ & $12(7.1)$ & $7(9.9)$ & $1(0.6)$ & \\
\hline Intermediate sarcosine type & $21(2.9)$ & $14(4.6)$ & $4(2.4)$ & $1(1.4)$ & $2(1.1)$ & \\
\hline Low sarcosine type & $219(30.4)$ & $93(30.7)$ & $54(32.0)$ & $26(36.6)$ & $46(25.8)$ & \\
\hline Null type & $445(61.7)$ & $180(59.4)$ & 99 (58.6) & $37(52.1)$ & $129(72.5)$ & \\
\hline
\end{tabular}

Bold number represents $\mathrm{p}<0.05$.

$\mathrm{TNBC}$, triple negative breast cancer. 
Table 4 Clinicopathologic characteristics of patients according to sarcosine metabolism type

\begin{tabular}{|c|c|c|c|c|c|c|}
\hline Parameter & $\begin{array}{l}\text { Total } \\
\mathrm{N}=721(\%)\end{array}$ & $\begin{array}{l}\text { High sarcosine type } \\
\mathrm{n}=36(\%)\end{array}$ & $\begin{array}{l}\text { Intermediate sarcosine type } \\
\mathrm{n}=\mathbf{2 1}(\%)\end{array}$ & $\begin{array}{l}\text { Low sarcosine type } \\
\mathrm{n}=\mathbf{2 1 9}(\%)\end{array}$ & $\begin{array}{l}\text { Null type } \\
\mathrm{n}=445(\%)\end{array}$ & $P$-value \\
\hline Age (years) & & & & & & 0.200 \\
\hline$\leq 50$ & $428(59.4)$ & $26(72.2)$ & $13(61.9)$ & $120(54.8)$ & $269(60.4)$ & \\
\hline$>50$ & $293(40.6)$ & $10(27.8)$ & $8(38.1)$ & $99(45.2)$ & $176(39.6)$ & \\
\hline Histologic grade & & & & & & 0.351 \\
\hline$|/| \mid$ & $485(67.3)$ & $29(80.6)$ & $13(61.9)$ & $147(67.1)$ & $296(66.5)$ & \\
\hline III & $236(32.7)$ & $7(19.4)$ & $8(38.1)$ & $72(32.9)$ & 149 (33.5) & \\
\hline Tumor stage & & & & & & 0.082 \\
\hline $\mathrm{T} 1$ & $355(49.2)$ & $12(33.3)$ & $10(47.6)$ & $120(54.8)$ & $213(47.9)$ & \\
\hline $\mathrm{T} 2 / \mathrm{T} 3$ & $366(50.8)$ & $24(66.7)$ & $11(52.4)$ & $99(45.2)$ & $232(52.1)$ & \\
\hline Nodal metastasis & & & & & & 0.799 \\
\hline Absent & $427(59.2)$ & $22(61.1)$ & $14(66.7)$ & $125(57.1)$ & $266(59.8)$ & \\
\hline Present & $294(40.8)$ & $14(38.9)$ & $7(33.3)$ & $94(42.9)$ & $179(40.2)$ & \\
\hline Estrogen receptor status & & & & & & 0.049 \\
\hline Negative & $259(35.9)$ & $9(25.0)$ & $3(14.3)$ & $75(34.2)$ & $172(38.7)$ & \\
\hline Positive & $462(64.1)$ & $27(75.0)$ & $18(85.7)$ & $144(65.8)$ & $273(61.3)$ & \\
\hline Progesterone receptor status & & & & & & 0.011 \\
\hline Negative & $347(48.1)$ & $13(36.1)$ & $4(19.0)$ & $102(46.6)$ & $228(51.2)$ & \\
\hline Positive & $374(51.9)$ & $23(63.9)$ & $17(81.0)$ & $117(53.4)$ & $217(48.8)$ & \\
\hline HER-2 status & & & & & & 0.072 \\
\hline Negative & $565(78.4)$ & $24(66.7)$ & $17(81.0)$ & $163(74.4)$ & $361(81.1)$ & \\
\hline Positive & $156(21.6)$ & $12(33.3)$ & $4(19.0)$ & $56(25.6)$ & $84(18.9)$ & \\
\hline Ki-67 LI (\%) & & & & & & 0.007 \\
\hline$\leq 14$ & $409(56.7)$ & $21(58.3)$ & $17(81.0)$ & $138(63.0)$ & $233(52.4)$ & \\
\hline$>14$ & $312(43.3)$ & $15(41.7)$ & $4(19.0)$ & $81(37.0)$ & $212(47.6)$ & \\
\hline Tumor recurrence & $63(8.7)$ & $8(22.2)$ & $1(4.8)$ & $15(6.8)$ & $39(8.8)$ & 0.022 \\
\hline No. of patient deaths & $61(8.5)$ & $5(13.9)$ & $1(4.8)$ & $20(9.1)$ & $35(7.9)$ & 0.558 \\
\hline
\end{tabular}

Bold number represents $\mathrm{p}<0.05$.

TNBC, triple negative breast cancer.

\section{Correlations between expression of sarcosine} metabolism-related proteins and clinicopathologic factors Analysis of sarcosine metabolism-related protein expression and clinicopathologic parameters showed an association between PR positivity and GNMT expression ( $\mathrm{p}=0.016)$, between HER-2 positivity and SARDH expression $(\mathrm{p}=0.016)$ and stromal PIPOX expression $(\mathrm{p}=0.004)$. Moreover, tumoral PIPOX expression was associated with lower Ki-67 LI ( $<$ 0.001, Table 5).

\section{Impact of expression of sarcosine metabolism-related} proteins on patient prognosis

To investigate the potential effects of sarcosine metabolismrelated protein expression on prognosis, univariate analysis was performed on all cases regardless of subtype. Factors associated with shorter disease-free survival (DFS) were GNMT expression $(\mathrm{p}=0.042)$, tumoral PIPOX negativity $(\mathrm{p}=0.039)$, and high sarcosine type $(\mathrm{p}=0.021$, Table 6 and
Figure 3). Multivariate Cox analysis revealed that higher $\mathrm{T}$ stage (hazard ratio: 2.123, 95\% CI: 1.167-3.861, $\mathrm{p}=0.014$ ), lymph node metastasis (hazard ratio: $2.344,95 \% \mathrm{CI}$ : 1.389 3.956, $\mathrm{p}=0.001$ ), and GNMT expression (hazard ratio: 2.408, 95\% CI: 1.154-5.024, p=0.019) were independent factors associated with shorter DFS. Additionally, higher T stage (hazard ratio: 1.829, 95\% CI: 1.028-3.255, $\mathrm{p}=0.040$ ) and lymph node metastasis (hazard ratio: 1.971, 95\% CI: 1.166-3.333, $\mathrm{p}=0.011$ ) were independent factors associated with shorter overall survival (OS) (Table 7).

The effect of sarcosine metabolism-related protein expression on prognosis according to molecular subtype was studied. In luminal $\mathrm{A}$, univariate analysis showed that SARDH expression was associated with shorter OS $(\mathrm{p}=0.010$, Figure 4$)$, and SARDH expression was an independent factor for shorter OS (hazard ratio: 3.793, 95\% CI: 1.231-11.68, $\mathrm{p}=0.020)$. In luminal $\mathrm{B}, \mathrm{GNMT}$ expression ( $p=0.003$ and 0.020 , respectively) and high 
Table 5 Correlations between the expression of sarcosine metabolism-related proteins and clinicopathologic parameters

\begin{tabular}{|c|c|c|c|c|c|c|c|c|c|c|c|c|}
\hline \multirow[t]{2}{*}{ Parameter } & \multicolumn{3}{|c|}{ GNMT } & \multicolumn{3}{|c|}{ SARDH } & \multicolumn{3}{|c|}{ PIPOX in tumor } & \multicolumn{3}{|c|}{ PIPOX in stroma } \\
\hline & $\begin{array}{c}\text { Negative } \\
\mathrm{n}=664 \\
(\%)\end{array}$ & $\begin{array}{c}\text { Positive } \\
n=57 \\
(\%)\end{array}$ & $P$-value* & $\begin{array}{c}\text { Negative } \\
\mathrm{n}=597 \\
(\%)\end{array}$ & $\begin{array}{c}\text { Positive } \\
\mathrm{n}=124 \\
(\%)\end{array}$ & $P$-value* & $\begin{array}{c}\text { Negative } \\
\mathrm{n}=570 \\
(\%)\end{array}$ & $\begin{array}{c}\text { Positive } \\
\mathrm{n}=151 \\
(\%)\end{array}$ & $P$-value* & $\begin{array}{c}\text { Negative } \\
\mathrm{n}=671 \\
(\%)\end{array}$ & $\begin{array}{c}\text { Positive } \\
\mathrm{n}=50 \\
(\%)\end{array}$ & $P$-value* \\
\hline Age (years) & & & 0.588 & & & 0.336 & & & 0.620 & & & 1.088 \\
\hline$\leq 50$ & 389 (58.6) & $39(68.4)$ & & $363(60.8)$ & $65(52.4)$ & & $346(60.7)$ & $82(54.3)$ & & $402(59.9)$ & $26(52.0)$ & \\
\hline$>50$ & $275(41.4)$ & 18 (31.6) & & $234(39.2)$ & $59(47.6)$ & & 224 (39.3) & $69(45.7)$ & & $269(40.1)$ & $24(48.0)$ & \\
\hline $\begin{array}{l}\text { Histologic } \\
\text { grade }\end{array}$ & & & 1.128 & & & 3.068 & & & 3.036 & & & 1.024 \\
\hline$|/| \mid$ & $443(66.7)$ & $42(73.7)$ & & $403(67.5)$ & $82(66.1)$ & & 385 (67.5) & $100(66.2)$ & & $455(67.8)$ & $30(60.0)$ & \\
\hline III & $221(33.3)$ & $15(26.3)$ & & $194(32.5)$ & $42(33.9)$ & & 185 (32.5) & $51(33.8)$ & & $216(32.2)$ & $20(40.0)$ & \\
\hline Tumor stage & & & 0.376 & & & 1.316 & & & 0.308 & & & 3.644 \\
\hline $\mathrm{T} 1$ & $333(50.2)$ & $22(38.6)$ & & $289(48.4)$ & 66 (53.2) & & $271(47.5)$ & $84(55.6)$ & & $330(49.2)$ & $25(50.0)$ & \\
\hline $\mathrm{T} 2 / \mathrm{T} 3$ & $331(49.8)$ & $35(61.4)$ & & 308 (51.6) & $58(46.8)$ & & 299 (52.5) & $67(44.4)$ & & $341(50.8)$ & $25(50.0)$ & \\
\hline $\begin{array}{l}\text { Nodal } \\
\text { metastasis }\end{array}$ & & & 2.116 & & & 3.640 & & & 2.604 & & & 2.524 \\
\hline Absent & $391(58.9)$ & $36(63.2)$ & & $353(59.1)$ & 74 (59.7) & & 340 (59.6) & $87(57.6)$ & & $399(59.5)$ & $28(56.0)$ & \\
\hline Present & $273(41.1)$ & $21(36.8)$ & & $244(40.9)$ & $50(40.3)$ & & $230(40.4)$ & $64(42.4)$ & & $272(40.5)$ & $22(44.0)$ & \\
\hline $\begin{array}{l}\text { Estrogen } \\
\text { receptor status }\end{array}$ & & & 0.060 & & & 3.644 & & & 1.672 & & & 2.132 \\
\hline Negative & $247(37.2)$ & $12(21.1)$ & & $215(36.0)$ & 44 (35.5) & & 209 (36.7) & $50(33.1)$ & & $239(35.6)$ & $20(40.0)$ & \\
\hline Positive & $417(62.8)$ & $45(78.9)$ & & $382(64.0)$ & $80(64.5)$ & & 361 (63.3) & $101(66.9)$ & & $432(64.4)$ & $30(60.0)$ & \\
\hline $\begin{array}{l}\text { Progesterone } \\
\text { eceptor status }\end{array}$ & & & 0.004 & & & 1.572 & & & 0.204 & & & 3.020 \\
\hline Negative & $330(49.7)$ & 17 (29.8) & & $283(47.4)$ & 64 (51.6) & & $285(50.0)$ & $62(41.1)$ & & $324(48.3)$ & $23(46.0)$ & \\
\hline Positive & 334 (50.3) & $40(70.2)$ & & 314 (52.6) & $60(48.4)$ & & $285(50.0)$ & 89 (58.9) & & $347(51.7)$ & $27(54.0)$ & \\
\hline HER-2 status & & & 0.876 & & & 0.004 & & & 3.072 & & & 0.001 \\
\hline Negative & $524(78.9)$ & 41 (71.9) & & $480(80.4)$ & 85 (68.5) & & 448 (78.6) & $117(77.5)$ & & $535(79.7)$ & $30(60.0)$ & \\
\hline Positive & $140(21.1)$ & $16(28.1)$ & & 117 (19.6) & 39 (31.5) & & $122(21.4)$ & $34(22.5)$ & & $136(20.3)$ & $20(40.0)$ & \\
\hline Ki-67 LI (\%) & & & 0.456 & & & 3.156 & & & $<0.001$ & & & 1.280 \\
\hline$\leq 14$ & $371(55.9)$ & $38(66.7)$ & & $340(57.0)$ & 69 (55.6) & & $303(53.2)$ & $106(70.2)$ & & $384(57.2)$ & $25(50.0)$ & \\
\hline$>14$ & $293(44.1)$ & 19 (33.3) & & $257(43.0)$ & $55(44.4)$ & & $267(46.8)$ & 45 (29.8) & & $287(42.8)$ & $25(50.0)$ & \\
\hline
\end{tabular}

Bold number represents $\mathrm{p}<0.05$.

*p-value was calculated by Bonferroni method.

sarcosine type ( $\mathrm{p}=0.002$ and 0.028 , respectively) were correlated with both shorter DFS and shorter OS (Figure 5), but no correlation was found in multivariate Cox analysis (Additional file 1: Table S2 and Additional file 1: Table S3).

\section{Discussion}

We investigated the expression of sarcosine metabolismrelated proteins in breast cancer, with a focus on molecular subtypes of breast cancer. GNMT, SARDH, and PIPOX showed the highest expression levels in HER-2 type cancer. Sarcosine has mostly been researched in prostate cancer; studies have shown that increased sarcosine level is associated with cancer progression, and in vitro and in vivo models have suggested a correlation between prostate cancer growth and progression [2]. Key results include the induction of invasive phenotypes by injection of sarcosine into benign prostate cells [1] and increased sarcosine levels in prostate cancer tissue [23]. Increased sarcosine levels in prostate cancer can be useful in cancer detection. Reports revealed that sarcosine had higher predictive value in detecting prostate cancer in tissue biopsy than PSA, particularly when PSA level was between 2 to $10 \mathrm{ng} / \mathrm{ml}$ or the gray zone [1], or when it was less than $4 \mathrm{ng} / \mathrm{ml}[24,25]$. Moreover, sarcosine levels and GNMT expression were also increased in tissue and feces of experimental azoxymethane-induced colorectal cancer [26].

Unfortunately, there is no prior research on sarcosine in breast cancers against which to compare our study 
Table 6 Univariate analysis of the impact of expression of serine/glycine metabolism-related proteins in breast cancers on disease-free survival and overall survival by the log-rank test

\begin{tabular}{|c|c|c|c|c|c|}
\hline \multirow[t]{2}{*}{ Parameter } & \multirow{2}{*}{$\begin{array}{l}\text { Number of patients/ } \\
\text { recurrence/death }\end{array}$} & \multicolumn{2}{|l|}{ Disease-free survival } & \multicolumn{2}{|l|}{ Overall survival } \\
\hline & & Mean survival $(95 \% \mathrm{Cl})$ months & $P$-value & Mean survival $(95 \% \mathrm{Cl})$ months & $P$-value \\
\hline GNMT & & & 0.042 & & 0.498 \\
\hline Negative & $664 / 54 / 55$ & $127(123-130)$ & & $129(127-132)$ & \\
\hline Positive & $57 / 9 / 6$ & $98(91-106)$ & & $126(116-135)$ & \\
\hline SARDH & & & 0.767 & & 0.264 \\
\hline Negative & $597 / 53 / 47$ & $126(122-130)$ & & $130(127-133)$ & \\
\hline Positive & $124 / 10 / 14$ & $121(115-127)$ & & $124(117-132)$ & \\
\hline PIPOX (T) & & & 0.039 & & 0.361 \\
\hline Negative & $570 / 57 / 52$ & $126(123-129)$ & & $129(126-131)$ & \\
\hline Positive & $151 / 6 / 9$ & $123(117-129)$ & & $131(126-135)$ & \\
\hline PIPOX (S) & & & 0.927 & & 0.636 \\
\hline Negative & $671 / 59 / 58$ & $126(122-130)$ & & $129(126-132)$ & \\
\hline Positive & $50 / 4 / 3$ & $122(114-130)$ & & $126(119-132)$ & \\
\hline Sarcosine metabolic type & & & 0.021 & & 0.503 \\
\hline High sarcosine type & $36 / 8 / 5$ & $90(79-101)$ & & $118(106-130)$ & \\
\hline Intermediate sarcosine type & $21 / 1 / 1$ & $108(102-114)$ & & $132(122-143)$ & \\
\hline Low sarcosine type & 219/15/20 & $120(115-125)$ & & $126(121-132)$ & \\
\hline Null type & $445 / 39 / 35$ & $127(124-131)$ & & $130(127-133)$ & \\
\hline
\end{tabular}

Bold number represents $p<0.05$.

findings. Reports indicate strong correlations between sarcosine metabolism-related proteins and prostate cancer. Compared to normal prostate tissue, prostate cancer tissue showed higher expression of GNMT, the sarcosine generating enzyme, and lower expression of SARDH and PIPOX, which are sarcosine metabolizing enzymes, suggesting a correlation between sarcosine level and the expression of sarcosine metabolism-related proteins [2]. Although we did not measure sarcosine levels directly from breast cancer tissues, we used expression levels of the major sarcosine metabolism-related proteins GNMT, SARDH, and PIPOX as surrogate for sarcosine level. The expression rate of GNMT, SARDH, and PIPOX were higher in HER-2 type relative to the others subtypes. Moreover, the proportion of high sarcosine type [GNMT $(+) /$ SARDH and PIPOX $(-)]$ was highest in HER-2 type tumors, suggesting that sarcosine levels are higher in HER-2 type breast cancers than in other molecular subtypes. The mechanism underlying higher expression of sarcosine metabolism-related protein in HER-2 type tumors
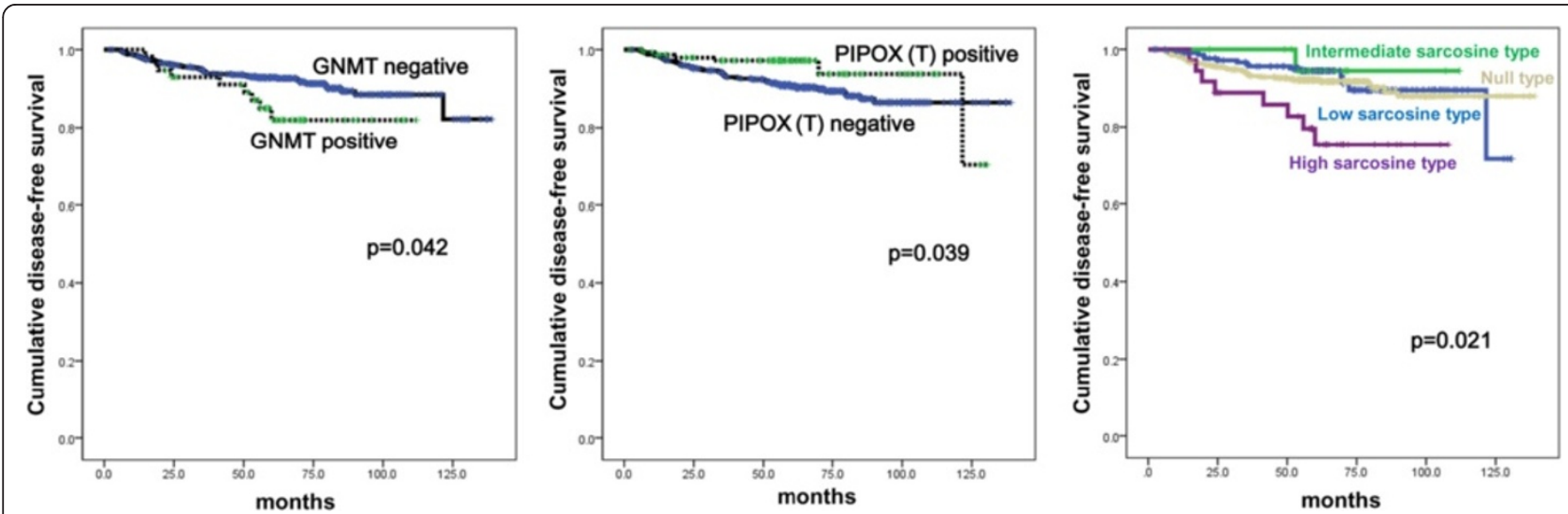

Figure 3 Disease-free survival and overall survival according to the expression of sarcosine metabolism-related proteins and sarcosine metabolism phenotype. 
Table 7 Multivariate analysis of breast-cancer survival

\begin{tabular}{|c|c|c|c|c|c|c|}
\hline \multirow[t]{2}{*}{ Included parameters } & \multicolumn{3}{|c|}{ Disease-free survival } & \multicolumn{3}{|c|}{ Overall survival } \\
\hline & Hazard ratio & $95 \% \mathrm{Cl}$ & $P$-value & Hazard ratio & $95 \% \mathrm{Cl}$ & $P$-value \\
\hline T stage & & & 0.014 & & & 0.040 \\
\hline $\mathrm{T} 1$ versus $\mathrm{T} 2-3$ & 2.123 & $1.167-3.861$ & & 1.829 & $1.028-3.255$ & \\
\hline N stage & & & 0.001 & & & 0.011 \\
\hline No versus N1-3 & 2.344 & $1.389-3.956$ & & 1.971 & $1.166-3.333$ & \\
\hline Histologic grade & & & 0.632 & & & 0.545 \\
\hline$|/| \mid$ versus III & 1.143 & $0.661-1.977$ & & 0.839 & $0.475-1.482$ & \\
\hline ER status & & & 0.111 & & & 0.079 \\
\hline Negative versus Positive & 1.795 & $0.874-3.686$ & & 1.888 & $0.929-3.836$ & \\
\hline PR status & & & 0.198 & & & 0.070 \\
\hline Negative versus Positive & 1.638 & $0.773-3.473$ & & 2.036 & $0.944-4.393$ & \\
\hline HER-2 status & & & 0.469 & & & 0.508 \\
\hline Negative versus Positive & 1.235 & $0.697-2.188$ & & 1.214 & $0.683-2.159$ & \\
\hline GNMT & & & 0.019 & & & 0.272 \\
\hline Negative versus Positive & 2.408 & $1.154-5.024$ & & 1.622 & $0.684-3.849$ & \\
\hline PIPOX (T) & & & 0.069 & & & 0.563 \\
\hline Negative versus Positive & 0.457 & $0.196-1.064$ & & 0.810 & $0.397-1.652$ & \\
\hline
\end{tabular}

Bold number represents $\mathrm{p}<0.05$.

requires elucidation in further validation studies. One hypothesis is that sarcosine, HER-2, and the androgen receptor influence each other. A previous study showed that sarcosine increased both HER-2 mRNA and protein levels in an androgen-dependent prostate cancer cell line, suggesting an association among the androgen receptor, HER-2, and sarcosine [27]. Correspondingly, the molecular apocrine type in breast cancer defined as ER-negative and AR-positive breast cancer showed significant overlap

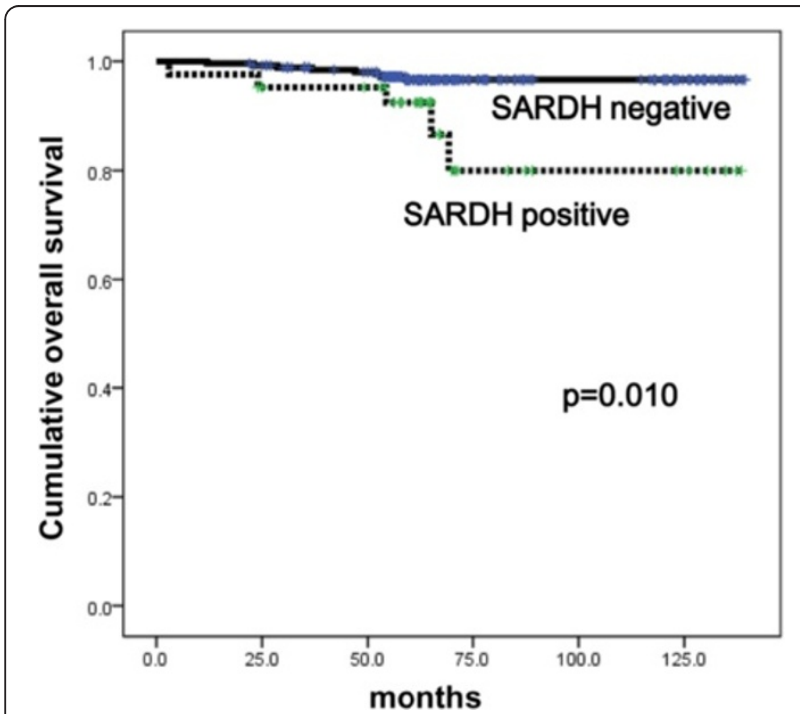

Figure 4 Overall survival according to the expression of SARDH in luminal A type breast cancer. with the HER-2-enriched group on gene profiling analysis [28]. Approximately 50\% of molecular apocrine type exhibited HER-2 amplification/overexpression, strongly implying a correlation between AR and HER-2. Further research on the effects of sarcosine on HER-2 according to AR status will help elucidate the mechanism of association among these key molecules in breast cancer. Another interesting finding is the correlation between GNMT positivity/PIPOX negativity and shorter DFS. A previous prostate cancer study revealed that increased levels of sarcosine induce invasion and intravasation [2], supporting the role of sarcosine as an oncometabolite in prostate cancer. A separate study showed that serum sarcosine levels were significantly higher in patients with metastatic prostate cancer [29]. It follows that sarcosine levels would be higher in breast cancer with positive GNMT and negative PIPOX, which could be the reason for shorter DFS in such breast cancer. Moreover, analysis of sarcosine metabolism phenotype revealed that patients with sarcosine-high type [GNMT(+)/SARDH and PIPOX(-)] tumors had a shorter DFS than patients with other breast cancer tumor types, suggesting that sarcosine level is a prognostic factor in breast cancer. A previous study reported a potential association between high GNMT cytoplasmic expression in prostate cancer and lower DFS rate [30], consistent with our findings. In contrast, research on hepatic cholangiocarcinoma suggests that GNMT expression was a favorable prognostic marker [31]. It is highly likely that sarcosine may have different roles according to the type and subtype of 

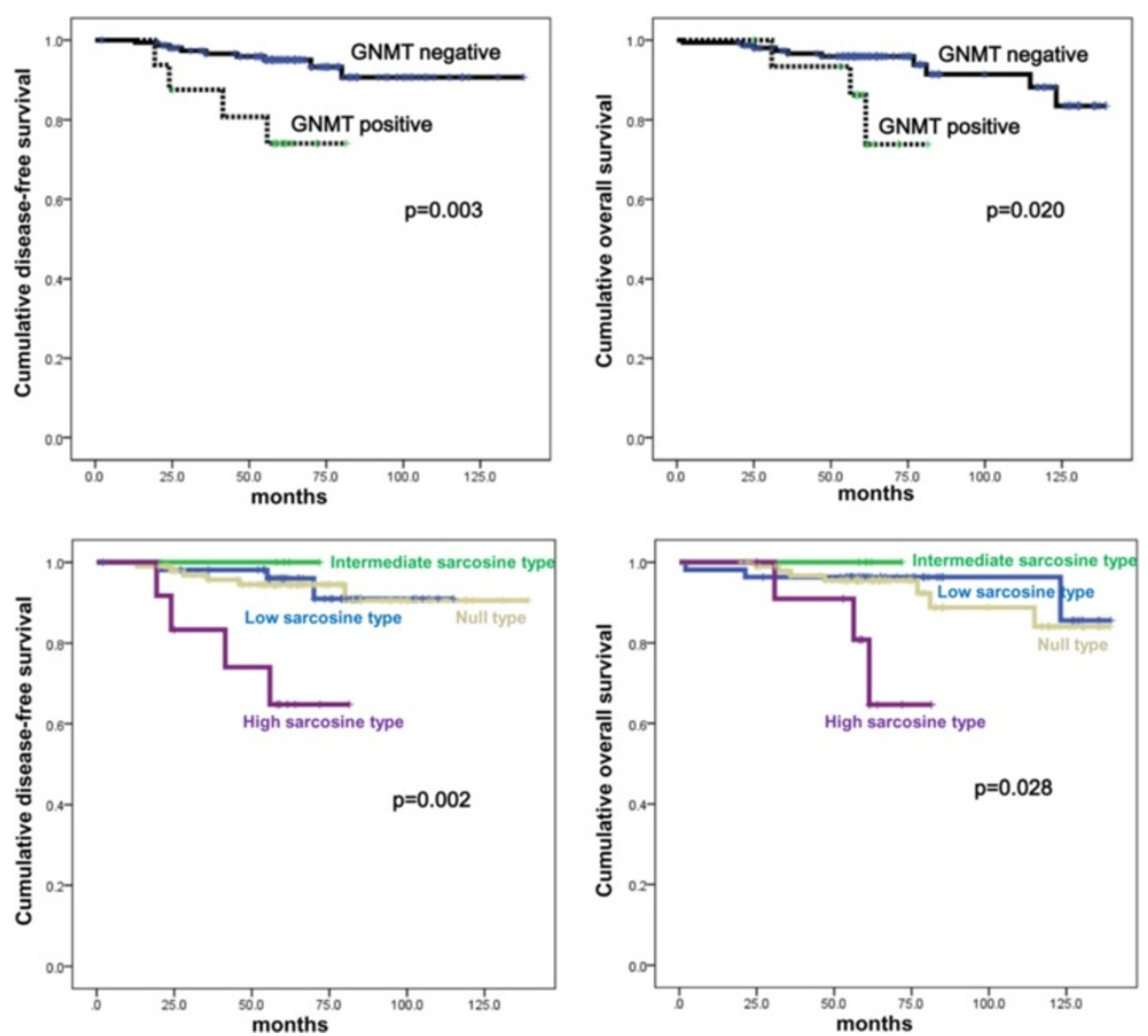

Figure 5 Disease-free survival and overall survival according to the expression of GNMT and sarcosine metabolism phenotype in luminal B type breast cancer.

cancer. Further validation studies to investigate the correlation between sarcosine level and cancer prognosis are necessary. That sarcosine metabolism-related proteins expression was significantly lower in TNBC-type tumors relative to the other subtypes was an unexpected result. TNBC tumors have highly aggressive histological characteristics such as high histologic grade, high levels of mitosis and tumor necrosis [7]. Accordingly, we expected TNBC tumor specimens to exhibit strong metabolic activity, as previous studies have reported increased expression of Glut-1 and CAIX in TNBC compared to other breast cancer subtypes $[8,9]$. Our results imply that sarcosine does not contribute significantly to the aggressiveness of TNBC. This topic requires further investigation.

\section{Conclusion}

The expressions of sarcosine metabolism-related proteins varied according to subtype of breast cancer; expression of these proteins was elevated in HER-2 type and decreased in TNBC. We also demonstrated that sarcosine metabolism-related proteins had prognostic utility in breast cancer patients.

\section{Additional file}

Additional file 1: Table S1. Source, clone, and dilutions of antibodies used in this study. Table S2. Multivariate analysis of breast-cancer survival in patients with luminal B type cancer. Table S3. Multivariate analysis of breast-cancer survival in patients with luminal A type cancer.

\section{Abbreviation}

SAM: S-adenosylmethionine; GNMT: Glycine N-methyltransferase; SARDH: Sarcosine-metabolizing enzymes - sarcosine dehydrogenase; PIPOX: I-pipecloioc acid oxidase; ER: Estrogen receptor; PR: Progesterone receptor; TNBC: Triple negative breast cancer; CAIX: Carbonic anhydrase; H\&E: Hematoxylin \& Eosin; ASCO: American society of clinical oncology; CAP: College of American Pathologists; DFS: Disease-free survival; OS: Overall survival.

\section{Competing interests}

The authors declare that they have no competing interests.

\section{Authors' contributions}

JKY participated in the design of the study and performed the statistical analysis and drafted the manuscript. DHK carried out the immunochemistry. JSK conceived the study, and participated in its design and coordination and helped to draft the manuscript. All authors read and approved the final manuscript.

\section{Acknowledgements}

This research was supported by the Basic Science Research Program through the National Research Foundation of Korea (NRF) funded by the Ministry of Education, Science and Technology (2012R1A1A1002886). This study was 
supported by a grant from National R\&D Program for Cancer Control, Ministry of Health \& Welfare, Republic of Korea (1420080).

Received: 13 March 2014 Accepted: 22 May 2014 Published: 28 May 2014

\section{References}

1. Sreekumar A, Poisson LM, Rajendiran TM, Khan AP, Cao Q, Yu J, Laxman B, Mehra R, Lonigro RJ, Li Y, Nyati MK, Ahsan A, Kalyana-Sundaram S, Han B, Cao X, Byun J, Omenn GS, Ghosh D, Pennathur S, Alexander DC, Berger A, Shuster JR, Wei JT, Varambally S, Beecher C, Chinnaiyan AM: Metabolomic profiles delineate potential role for sarcosine in prostate cancer progression. Nature 2009, 457:910-914.

2. Khan AP, Rajendiran TM, Ateeq B, Asangani IA, Athanikar JN, Yocum AK, Mehra R, Siddiqui J, Palapattu G, Wei JT, Michailidis G, Sreekumar A, Chinnaiyan AM: The role of sarcosine metabolism in prostate cancer progression. Neoplasia 2013, 15:491-501.

3. Baum CE, Price DK, Figg WD: Sarcosine as a potential prostate cancer biomarker and therapeutic target. Cancer Biol Ther 2010, 9:341-342.

4. Cernei N, Heger Z, Gumulec J, Zitka O, Masarik M, Babula P, Eckschlager T, Stiborova M, Kizek R, Adam V: Sarcosine as a potential prostate cancer biomarker-a review. Int J Mol Sci 2013, 14:13893-13908.

5. Perou CM, Sørlie T, Eisen MB, van de Rijn M, Jeffrey SS, Rees CA, Pollack JR, Ross DT, Johnsen H, Akslen LA, Fluge O, Pergamenschikov A, Williams C, Zhu SX, Lønning PE, Børresen-Dale AL, Brown PO, Botstein D: Molecular portraits of human breast tumours. Nature 2000, 406:747-752.

6. Sørlie T, Perou CM, Tibshirani R, Aas T, Geisler S, Johnsen H, Hastie T, Eisen $M B$, van de Rijn M, Jeffrey SS, Thorsen T, Quist H, Matese JC, Brown PO, Botstein D, Lønning PE, Børresen-Dale AL: Gene expression patterns of breast carcinomas distinguish tumor subclasses with clinical implications. Proc Natl Acad Sci U S A 2001, 98:10869-10874.

7. Reis-Filho JS, Tutt AN: Triple negative tumours: a critical review. Histopathology 2008, 52:108-118.

8. Pinheiro C, Sousa B, Albergaria A, Paredes J, Dufloth R, Vieira D, Schmitt F, Baltazar F: GLUT1 and CAIX expression profiles in breast cancer correlate with adverse prognostic factors and MCT1 overexpression. Histol Histopathol 2011, 26:1279-1286.

9. Choi J, Jung WH, Koo JS: Metabolism-related proteins are differentially expressed according to the molecular subtype of invasive breast cancer defined by surrogate immunohistochemistry. Pathobiology 2013, 80:41-52.

10. Kim S, Kim do H, Jung WH, Koo JS: Expression of glutamine metabolismrelated proteins according to molecular subtype of breast cancer. Endocr Relat Cancer 2013, 20:339-348.

11. Tulinius $H$, Egilsson V, Olafsdottir GH, Sigvaldason H: Risk of prostate, ovarian, and endometrial cancer among relatives of women with breast cancer. BMJ 1992, 305:855-857.

12. Sellers TA, Potter JD, Rich SS, Drinkard CR, Bostick RM, Kushi LH, Zheng W Folsom AR: Familial clustering of breast and prostate cancers and risk of postmenopausal breast cancer. J Natl Cancer Inst 1994, 86:1860-1865.

13. Zhu X, Daffada AA, Chan CM, Dowsett M: Identification of an exon 3 deletion splice variant androgen receptor mRNA in human breast cancer. Int J Cancer 1997, 72:574-580.

14. Ford D, Easton DF, Bishop DT, Narod SA, Goldgar DE: Risks of cancer in BRCA1-mutation carriers. Breast Cancer Linkage Consortium. Lancet 1994, 343:692-695.

15. Struewing JP, Hartge P, Wacholder S, Baker SM, Berlin M, McAdams M, Timmerman MM, Brody LC, Tucker MA: The risk of cancer associated with specific mutations of BRCA1 and BRCA2 among Ashkenazi Jews. N Engl J Med 1997, 336:1401-1408.

16. Diamandis EP, Yu H, Sutherland DJ: Detection of prostate-specific antigen immunoreactivity in breast tumors. Breast Cancer Res Treat 1994, 32:301-310.

17. Yu H, Giai M, Diamandis EP, Katsaros D, Sutherland DJ, Levesque MA Roagna $R$, Ponzone $R$, Sismondi P: Prostate-specific antigen is a new favorable prognostic indicator for women with breast cancer. Cancer Res 1995, 55:2104-2110.

18. Elston CW, Ellis IO: Pathological prognostic factors in breast cancer. I. The value of histological grade in breast cancer: experience from a large study with long-term follow-up. Histopathol 1991, 19:403-410.
19. Hammond ME, Hayes DF, Dowsett M, Allred DC, Hagerty KL, Badve S, Fitzgibbons PL, Francis G, Goldstein NS, Hayes M, Hicks DG, Lester S, Love R, Mangu PB, McShane L, Miller K, Osborne CK, Paik S, Perlmutter J, Rhodes A, Sasano H, Schwartz JN, Sweep FC, Taube S, Torlakovic EE, Valenstein P, Viale G, Visscher D, Wheeler T, Williams RB, Wittliff JL, Wolff AC: American Society of Clinical Oncology/College Of American Pathologists guideline recommendations for immunohistochemical testing of estrogen and progesterone receptors in breast cancer. J Clin Oncol 2010, 28:2784-2795.

20. Wolff AC, Hammond ME, Schwartz JN, Hagerty KL, Allred DC, Cote RJ, Dowsett M, Fitzgibbons PL, Hanna WM, Langer A, McShane LM, Paik S, Pegram MD, Perez EA, Press MF, Rhodes A, Sturgeon C, Taube SE, Tubbs R, Vance GH, van de Vijver M, Wheeler TM, Hayes DF: American Society of Clinical Oncology/College of American Pathologists guideline recommendations for human epidermal growth factor receptor 2 testing in breast cancer. J Clin Oncol 2007, 25:118-145.

21. Won KY, Kim GY, Kim YW, Song JY, Lim SJ: Clinicopathologic correlation of beclin-1 and bcl-2 expression in human breast cancer. Hum Pathol 2010, 41:107-112.

22. Goldhirsch A, Wood WC, Coates AS, Gelber RD, Thurlimann B, Senn HJ: Strategies for subtypes-dealing with the diversity of breast cancer: highlights of the St. Gallen International Expert Consensus on the Primary Therapy of Early Breast Cancer 2011. Ann Oncol 2011, 22:1736-1747

23. Jentzmik F, Stephan C, Lein M, Miller K, Kamlage B, Bethan B, Kristiansen G, Jung $K$ : Sarcosine in prostate cancer tissue is not a differential metabolite for prostate cancer aggressiveness and biochemical progression. J Urol 2011, 185:706-711.

24. Lucarelli G, Fanelli M, Larocca AM, Germinario CA, Rutigliano M, Vavallo A, Selvaggi FP, Bettocchi C, Battaglia M, Ditonno P: Serum sarcosine increases the accuracy of prostate cancer detection in patients with total serum PSA less than $4.0 \mathrm{ng} / \mathrm{ml}$. Prostate 2012, 72:1611-1621.

25. Lucarelli G, Rutigliano M, Bettocchi C, Palazzo S, Vavallo A, Galleggiante V, Trabucco S, Di Clemente D, Selvaggi FP, Battaglia M, Ditonno P: Spondin-2, a secreted extracellular matrix protein, is a novel diagnostic biomarker for prostate cancer. J Urol 2013, 190:2271-2277.

26. Montrose DC, Zhou XK, Kopelovich L, Yantiss RK, Karoly ED, Subbaramaiah K, Dannenberg AJ: Metabolic profiling, a noninvasive approach for the detection of experimental colorectal neoplasia. Cancer Prev Res (Phila) 2012, 5:1358-1367.

27. Dahl M, Bouchelouche P, Kramer-Marek G, Capala J, Nordling J, Bouchelouche $\mathrm{K}$ : Sarcosine induces increase in HER2/neu expression in androgendependent prostate cancer cells. Mol Biol Rep 2011, 38:4237-4243.

28. Farmer $P$, Bonnefoi $H$, Becette $V$, Tubiana-Hulin M, Fumoleau P, Larsimont D, Macgrogan G, Bergh J, Cameron D, Goldstein D, Duss S, Nicoulaz AL, Brisken C, Fiche M, Delorenzi M, Iggo R: Identification of molecular apocrine breast tumours by microarray analysis. Oncogene 2005, 24:4660-4671.

29. Lucarelli G, Ditonno P, Bettocchi C, Spilotros M, Rutigliano M, Vavallo A, Galleggiante V, Fanelli M, Larocca AM, Germinario CA, Maiorano E, Selvaggi FP, Battaglia M: Serum sarcosine is a risk factor for progression and survival in patients with metastatic castration-resistant prostate cancer. Future Oncol 2013, 9:899-907.

30. Song $Y H$, Shiota M, Kuroiwa K, Naito S, Oda Y: The important role of glycine $\mathrm{N}$-methyltransferase in the carcinogenesis and progression of prostate cancer. Mod Pathol 2011, 24:1272-1280.

31. Huang YC, Chen M, Shyr YM, Su CH, Chen CK, Li AF, Ho DM, Chen YM: Glycine N-methyltransferase is a favorable prognostic marker for human cholangiocarcinoma. J Gastroenterol Hepatol 2008, 23:1384-1389.

doi:10.1186/1479-5876-12-149

Cite this article as: Yoon et al:: Implications of differences in expression of sarcosine metabolism-related proteins according to the molecular subtype of breast cancer. Journal of Translational Medicine 2014 12:149. 